\title{
Review of: "Wolbachia endosymbionts in two Anopheles species indicates independent acquisitions and lack of prophage elements"
}

Michaël Luciano Tantely

Potential competing interests: The author(s) declared that no potential competing interests exist.

This interesting manuscript will worth publication after minor revision.

1) Before acceptation of the paper, double-check the abbreviation of genus Anopheles throught of the manuscript (for exemple, use "An. gambiae" instead of "A. gambiae", idem for A. demeilloni, A. moucheti,

A. funestus

2) The manuscript work on several complex species (An. gambiae and An. marshalli) and group of species (An. funestus). A special discussion on these complex and group should be added. For exemple, is A. marshalli you call here is the species of the marshalli complex or the main complexe?

3) line 188 "lower" or "decreased"? 